\title{
Comparison of irradiated and non-irradiated acellular dermal matrices in breast reconstruction under radiotherapy
}

\author{
Soo Jin Woo ${ }^{1}$, Jeong Hyun $\mathrm{Ha}^{2}$, Ung Sik Jin ${ }^{1}$ \\ ${ }^{1}$ Department of Plastic and Reconstructive Surgery, Seoul National University College of Medicine, Seoul; ${ }^{2}$ Department of Plastic and \\ Reconstructive Surgery, Seoul Metropolitan Government-Seoul National University Boramae Medical Center, Seoul, Korea
}

Background Acellular dermal matrices (ADMs) have become an essential material for implant-based breast reconstruction. No previous studies have evaluated the effects of sterility of ADM under conditions of radiation. This study compared sterile (irradiated) and aseptic (non-irradiated) ADMs to determine which would better endure radiotherapy.

Methods Eighteen male Balb/C mice were assigned to the control group with no irradiation (group 1) or one of two other groups with a radiation intensity of $10 \mathrm{~Gy}$ (group 2) or $20 \mathrm{~Gy}$ (group 3). Both sterile and aseptic ADMs were inserted into the back of each mouse. The residual volume of the ADM (measured using three-dimensional photography), cell incorporation, $\alpha$-smooth muscle actin expression, and connective tissue growth factor expression were evaluated. The thickness and CD3 expression of the skin were measured 4 and 8 weeks after radiation.

Results In groups 2 and 3, irradiated ADMs had a significantly larger residual volume than the non-irradiated $A D M s$ after 8 weeks $(P<0.05)$. No significant differences were found in cell incorporation and the amount of fibrosis between irradiated and non-irradiated ADMs. The skin was significantly thicker in the non-irradiated ADMs than in the irradiated ADMs in group $3(P<0.05)$. CD3 staining showed significantly fewer inflammatory cells in the skin of irradiated ADMs than in non-irradiated ADMs in all three groups after 4 and 8 weeks $(\mathrm{P}<0.05)$.

Conclusions Under radiation exposure, irradiated ADMs were more durable, with less volume decrease and less deposition of collagen fibers and inflammatory reactions in the skin than in non-irradiated ADMs.

Keywords Acellular dermal matrix / Radiotherapy / Breast reconstruction
Correspondence: Ung Sik Jin Department of Plastic and Reconstructive Surgery, Seoul National University College of Medicine, 101 Daehak-ro, Jongno-gu, Seoul 03080, Korea

Tel: +82-2-2072-3642

Fax: +82-2-3675-7792

E-mail: usj1011@snu.ac.kr

Received: July 23, 2020 • Revised: October 20, $2020 \bullet$ Accepted: November 3, 2020

pISSN: 2234-6163 • elSSN: 2234-6171 • https://doi.org/10.5999/aps.2020.01522• Arch Plast Surg 2021;48:33-43

\section{INTRODUCTION}

Acellular dermal matrices (ADMs) have become an essential component in expander- and implant-based breast reconstruc- tion due to their numerous benefits [1-3]. Even after preoperative or postoperative radiotherapy, $\mathrm{ADMs}$ do not appear to be associated with adverse effects or increased complications, such as capsular contracture, infection, and implant loss beyond what

Copyright $(\odot 2021$ The Korean Society of Plastic and Reconstructive Surgeons

This is an Open Access article distributed under the terms of the Creative Commons Attribution Non-Commercial License (https://creativecommons.org/

licenses/by-nc/4.0/) which permits unrestricted non-commercial use distribution, and reproduction in any medium, provided the original work is properly cited.

www.e-aps.org 
would be expected with radiotherapy alone [4-6].

The human ADMs currently available for breast reconstruction are either sterile or aseptic. The production of aseptic ADMs involves proprietary semi-contained processes such as using detergents and antibiotics to wash the allograft, as well as mechanical processing steps that lead to near-complete decellularization and decontamination [7]. However, to make a sterile $\mathrm{ADM}$, it is mandatory to perform additional validated terminal sterilization processes following initial cleansing and decellularization. Gamma radiation, electron beam radiation, or chemical solutions are used for terminal sterilization. The different ADM processing techniques may cause disparities in structural and biomechanical properties, further affecting the surgical outcomes.

Theoretically, using a sterile $\mathrm{ADM}$ would be expected to reduce the risk of infection in breast reconstruction. However, limited studies in the literature have compared sterile ADMs to aseptic $\mathrm{ADMs}$ in breast reconstruction, with mixed results $[8,9]$. Moreover, no published studies have investigated the effects of diverse $\mathrm{ADMs}$ in patients undergoing radiotherapy. Radiotherapy has become integral for breast cancer treatment in patients with both early and late-stage disease. While radiotherapy increases the risk of complications in implant-based breast reconstruction [10], ADM may reduce the adverse effects of radiotherapy on reconstructed breasts [4]. The aim of this study was to predict the possible impacts of ADM sterility on implantbased breast reconstruction with radiation exposure. In this experiment, we compared ADMs with different processing techniques to determine the optimal method for enabling $\mathrm{ADMs}$ to endure the adverse effects of radiotherapy. The authors hypothesized that irradiated, sterile ADMs would show less structural changes and inflammatory response under radiation than nonirradiated, aseptic ADMs.

\section{METHODS}

\section{Pilot experiment for setting the radiation dose}

The Animal Care and Utilization Committee of the Korea Institute of Radiological and Medical Sciences approved all procedures (approval No. KIRAMS 2018-0078). The preliminary experiment was designed to evaluate the amount of skin damage in response to increasing doses of radiation and to determine the maximal dose of radiation that would not induce structural changes in the skin (Fig. 1).

Five male Balb/C mice (average weight, $20 \mathrm{~g}$ ) were randomly selected (day 0 ). The mice had 1 week of acclimatization following transportation to restore homeostasis. On day 7, all mice were anesthetized through intraperitoneal injections of $100 \mathrm{mg} /$ $\mathrm{kg}$ of ketamine and $10 \mathrm{mg} / \mathrm{kg}$ of xylazine solution. After confirming that an adequate depth of anesthesia had been achieved, a lead shield was placed on each animal to cover the vertebral area. The mouse was placed $80 \mathrm{~cm}$ from the gamma radiation source, which was operated using settings of $225 \mathrm{kV}$ and $13 \mathrm{~mA}$ with a 0.2-mm copper filter and a $136.21 \mathrm{cGy} / \mathrm{min}$ dose rate (Gammabeam 100-80; Best Theratronics Ltd., Seoul, Korea). The radiation dose was 0 (sham), $10 \mathrm{~Gy}, 20 \mathrm{~Gy}, 30 \mathrm{~Gy}$, and $40 \mathrm{~Gy}$, respectively, for each of the five mice. On day 21 , skin from the back of each mouse was harvested using scissors and fixed for a 48-hour

\section{Fig. 1. Schematic diagram of the experiment}

A pilot experiment was done with five male Balb/C mice to establish a radiation dose that does not disrupt the structures of the skin. Based on the results, radiation intensities of $20 \mathrm{~Gy}$ and less were used for the main experiment. ADM, acellular dermal matrix.

Pilot experiment for setting the radiation dose
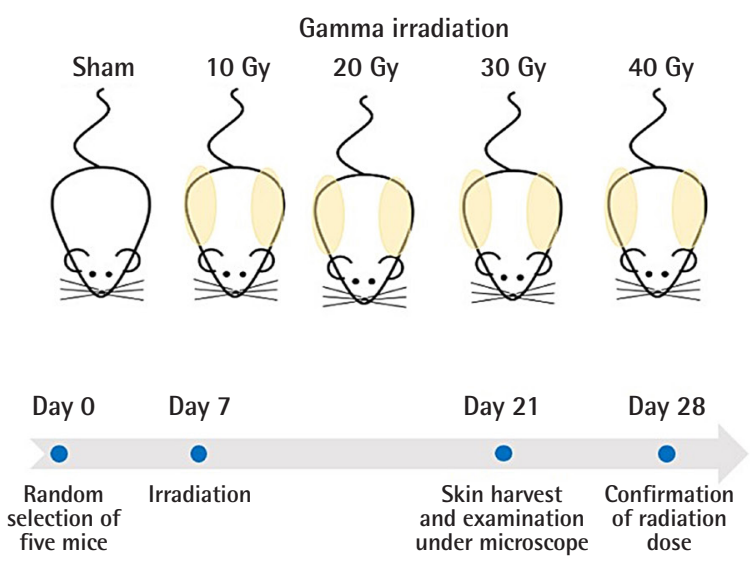

\section{Main experiment}

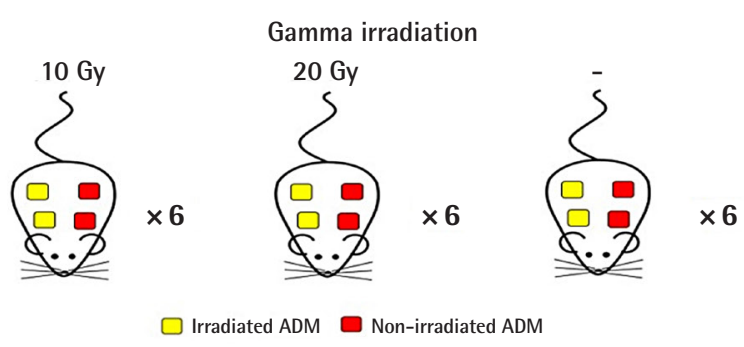

\begin{tabular}{|c|c|c|c|c|c|}
\hline Day 0 & Day 7 & Day 14 & Day 19 & 4th week & 8th week \\
\hline 0 & 0 & 0 & - & - & - \\
\hline $\begin{array}{l}\text { Assigning } \\
\text { mice into } \\
\text { three groups } \\
\text { of six animals }\end{array}$ & $\begin{array}{l}\text { Insertion } \\
\text { of ADM }\end{array}$ & $\begin{array}{l}10 \mathrm{~Gy} \\
\text { irradiation } \\
\text { for groups } \\
2 \text { and } 3\end{array}$ & $\begin{array}{l}10 \mathrm{~Gy} \\
\text { irradiation } \\
\text { for group } 3\end{array}$ & $\begin{array}{l}\text { Harvest of } \\
\text { 4th week } \\
\text { samples }\end{array}$ & $\begin{array}{c}\text { Harvest of } \\
\text { 8th week } \\
\text { samples }\end{array}$ \\
\hline
\end{tabular}


period in a $10 \%$ formalin solution. After fixation, the skin was embedded in paraffin, and 5- $\mu \mathrm{m}$ sections were cut and stained with hematoxylin and eosin $(\mathrm{H} \& \mathrm{E})$. Using $\times 400$ magnification under an Olympus BX43 microscope (Olympus, Center Valley, PA, USA), normal skin structure was observed. Structural changes involving the disruption of layered collagen were found in the dermis of mice with radiation intensities over $20 \mathrm{~Gy}$.

\section{Main experiment}

Eighteen male Balb/C mice (average weight, $21 \mathrm{~g}$ ) were randomly assigned to one of three groups of six animals on day 0 . The ADMs were inserted on day 7. One week after insertion (day 14), group 2 (10 Gy) and group 3 (20 Gy) were irradiated with an intensity of $10 \mathrm{~Gy}$. Group 1 (0 Gy) was not irradiated and served as a control group. Five days later (day 19), group 3 $(20 \mathrm{~Gy})$ was irradiated with an additional $10-\mathrm{Gy}$ dose of radiation, adding up to a total dose of $20 \mathrm{~Gy}$.

All ADMs (L\&C Bio, Seongnam, Korea) were from donated human skin manufactured using different sterilization methods. Non-irradiated, aseptic ADMs were manufactured by washing them with $70 \%$ alcohol and cleansing with sterile saline. Irradiated, sterile ADMs (MegaDerm; L\&C Bio) went through gamma radiation treatment to eliminate viruses, bacteria, and spores, achieving a $10^{-6}$ sterility level.

Aseptic $\mathrm{ADM}$ and sterile $\mathrm{ADM}$ were cut to dimensions of $0.5 \times 0.5 \mathrm{~cm}$ and a $0.2-\mathrm{cm}$ thickness and kept moist in normal saline solution. The experimental animals were anesthetized using intraperitoneal injections of a mixture of $100 \mathrm{mg} / \mathrm{kg}$ of ketamine and $10 \mathrm{mg} / \mathrm{kg}$ of xylazine solution. After confirming that anesthesia was adequate, four $1 \times 1 \mathrm{~cm}$ patches, one on each of the four quadrants of the back, were shaved, and the area was cleaned with povidone-iodine solution. On each shaved site, an incision of $0.5-1 \mathrm{~cm}$ was made with a no. 15 scalpel blade. Dissection at the subcutaneous level was performed to make a subdermal pouch for insertion of the ADM. Two sterile and two aseptic $\mathrm{ADMs}$ were inserted in four pouches in every mouse. The wound was closed with interrupted 4-0 polyglyconate sutures. Aseptic dressing with antibiotic ointment and gauze followed.

On day 14, the animals were anesthetized, and the backs of each of the animals in group 2 (10 Gy) and group 3 (20 Gy) were irradiated with a single dose of $10 \mathrm{~Gy}$, using a gamma beam operated at $225 \mathrm{kV}$ and $13 \mathrm{~mA}$ with a 0.2 -mm copper filter. The mice were kept inside an acrylic box filtered to outside air and were restrained with limbs extended with tape. The grafted area was covered with a $0.5-\mathrm{cm}$ bolus, and the collimator was positioned over the $\mathrm{ADM}$ area. On day 19, an additional 10-Gy radiation dose was administered to the mice in group 3 (20 Gy) using the same procedure as described above.

Three mice were sacrificed 4 weeks after irradiation from each group, while the other three were sacrificed 8 weeks after irradiation. From each mouse, two sterile and two aseptic ADMs were harvested with four $1-\mathrm{cm}$ incisions on four pouches at the back.

\section{Volume changes under the 3D camera}

A three-dimensional (3D) digital camera system (Antera 3D Camera; Miravex, Dublin, Ireland) was used for volumetric measurements. Since the harvested ADMs had curvy margins and were not uniformly square, the 3D camera made it possible to obtain accurate and objective quantifications of the samples' volumes. For all three groups, pictures of the ADM were taken with the 3D camera before insertion and after 4 and 8 weeks. The volume was measured as a percentage relative to the volume immediately before insertion, which was represented as $100 \%$ (day 7 ).

\section{Histologic evaluation}

\section{(1) H\&E staining}

Samples of ADMs were harvested and fixed in 10\% formalin solution for 24 hours. After fixation, samples were embedded in paraffin, and 5- $\mu \mathrm{m}$ sections were cut and stained with H\&E to examine their histopathological characteristics. With the Olympus BX43 microscope, the structure of the ADMs and cell incorporation were evaluated under $\times 12.5$ and $\times 400$ magnification. The sections stained with H\&E were used to determine the fibroblast count under $\times 400$ magnification. Four areas were analyzed for each specimen (two at the center of the sample and two at the periphery), and none of the areas overlapped with the other in order to provide a true nucleus count. The nuclei of the cells were quantified as the total number per high-power field.

\section{(2) Masson trichrome staining of collagen fibers}

Another set of skin samples were stained with Masson trichrome after fixation in a $10 \%$ formalin solution for 24 hours. Samples were sectioned into 5- $\mu \mathrm{m}$ sections. The collagen fibers were stained in blue, the nuclei in black, and the background in red. The thickness of the skin from the MT-stained samples was obtained from photos under $\times 100$ magnification.

(3) Immunohistochemistry of alpha-smooth muscle actin and $\mathrm{CD} 3$

Human dermal myofibroblasts, characterized by the expression of alpha-smooth muscle actin ( $\alpha$-SMA), are considered to be involved in contractile force generation during normal wound healing and pathological contracture. $\alpha$-SMA is an actin isoform that plays an important role in fibrogenesis and can be found in 
smooth muscle cells, myofibroblasts, and blood vessels. CD3 is a protein complex and $\mathrm{T}$ cell co-receptor that activates cytotoxic $\mathrm{T}$ cells and $\mathrm{T}$ helper cells. It is bound to the membranes of all mature $\mathrm{T}$ cells, but in essentially no other cell type; therefore, $\mathrm{CD} 3$ is a useful immunohistochemical marker of $\mathrm{T}$ cells in tissue sections.

For immunohistochemical analyses of $\alpha$-SMA and CD3, paraffin-embedded tissues were used with the $\alpha$-SMA antibody (ab5694; Abcam, Cambridge, MA, USA) and the CD3 antibody (ab5690; Abcam). The 5- $\mu \mathrm{m}$ paraffin sections were placed on poly-L-lysine-coated slides. After deparaffinization, endogenous peroxidase was reduced by incubating sections with 3\% hydrogen peroxidase in phosphate-buffered saline for 5 minutes. The secondary antibodies used were the Dako REAL EnVision Detection System, peroxidase/DAB+, rabbit/mouse (k5007; Dako, Santa Clara, CA, USA). Diaminobenzidine was used as a chromogen. Hematoxylin was used for counterstaining.

Images were captured using $\times 40$ magnification for $\alpha$-SMA by light microscopy. After being uploaded to a computer, all photos were opened with ImageJ, a Java program for image processing. The ruler at the base of the image included in the frame was used to calibrate the measurements of each image. The outline of the stained area was defined from the photographic image using a digital pad. After tracing, the ImageJ software calculated the stained area of $\alpha$-SMA and reported it as the percent area. The percentage of CD3-positive cells was calculated based on a manual count under $\times 400$ magnification by light microscopy.

\section{(4) Quantitative real-time RT-PCR of CTGF}

Connective tissue growth factor (CTGF) is a member of the CCN (CTGF/CYR61/NOV) family of novel cysteine-rich, secreted proteins. CTGF stimulates the proliferation, chemotaxis, and adhesion of connective tissue cells. Transforming growth factor-beta (TGF- $\beta$ ) rapidly induces CTGF expression in skin fibroblasts, and recent studies have found evidence that CTGF mediates the inductive effects of TGF- $\beta$ on procollagen production [11]. Therefore, CTGF is thought to be an essential mediator of fibrosis. Total RNA was extracted using TRizol from transplanted ADMs, and CDNA was synthesized by Maxime RT PreMix (oligo dT primer) (iNtRON Biotechnology, Inc., Seongnam, Korea). Reverse-transcription polymerase chain reaction (RT-PCR) reactions were performed using EvaGreen QMaster Kit (Cosmogenetech, Seoul, Korea). The RT-PCR assay was performed to detect the CTGF gene expression using CTGF receptor primers: forward, 5'-TGCGAAGCTGACCTGGAGGAAA-3' and reverse, 5' -CCGCAGAACTTAGCCCTGTATGTATG-3'.

\section{Statistical analysis}

Experimental values were presented as the median and interquartile range. Statistical significance was determined via the Mann-Whitney U test for comparisons between the two groups. Statistical analyses were performed using PASW statistics 18 (SPSS; IBM Corp., Armonk, NY, USA). P-values $<0.05$ were considered to indicate statistical significance.

\section{RESULTS}

\section{Volume change of ADM}

A volumetric evaluation was performed to confirm the durability of $\mathrm{ADM}$ under radiation. In group 1 (0 Gy), there was no significant difference in residual volume between the non-irradiated $\mathrm{ADM}$ and the irradiated ADM. In both groups 2 (10 Gy) and $3(20 \mathrm{~Gy})$, the residual volume was significantly larger in the irradiated $\mathrm{ADM}$ than in the non-irradiated $\mathrm{ADM}$ after 8 weeks $(\mathrm{P}<0.05)$ (Fig. 2).

\section{Fibrosis of ADM}

A comparison of the nucleus count of cells in ADMs after 4 weeks and 8 weeks revealed no significant differences in the amount of incorporation in the irradiated and non-irradiated ADMs in any group (Fig. 3). Regarding myofibroblasts, which play a central role in tissue fibrosis, there were no significant differences in the expression of $\alpha$-SMA between the irradiated and non-irradiated ADMs in any group (Fig. 4). The RT-PCR results also indicated that the level of mRNA of CTGF, an important mediator of fibrosis, was similar in the irradiated and nonirradiated ADMs and showed no significant differences (Fig. 5).

\section{Fibrosis and inflammation of the skin}

There was no visible change in the skin of all mice in the three groups. The collagen structure was observed done under Masson trichrome staining. The thickness of the skin of the non-irradiated $\mathrm{ADMs}$ was significantly greater than that of irradiated $\mathrm{ADMs}$ in group $3(20 \mathrm{~Gy})$ after 4 and 8 weeks $(\mathrm{P}<0.05)$. No significant differences were found in group 1 (0 Gy) or group 2 (10 Gy) (Fig. 6). CD3 staining showed a significant difference in inflammatory cells in irradiated ADMs compared to non-irradiated $\mathrm{ADMs}$ in all three groups after 4 and 8 weeks $(\mathrm{P}<0.05)$. There was a significantly smaller quantity of biomarkers of inflammation in irradiated ADMs than in non-irradiated ADMs (Fig. 7).

\section{DISCUSSION}

In recent years, $\mathrm{ADMs}$ have been used in more than $60 \%$ of 


\section{Fig. 2. Residual volume of ADM}

Volumetric measurements with the aid of a three-dimensional (3D) camera showed that the residual volume was significantly larger in irradiated acellular dermal matrix (ADM) than in non-irradiated ADM in groups 2 and 3 after 8 weeks. (A) 3D camera images of ADMs. (B) Quantification of residual volume of $A D M .{ }^{a} P<0.05$.
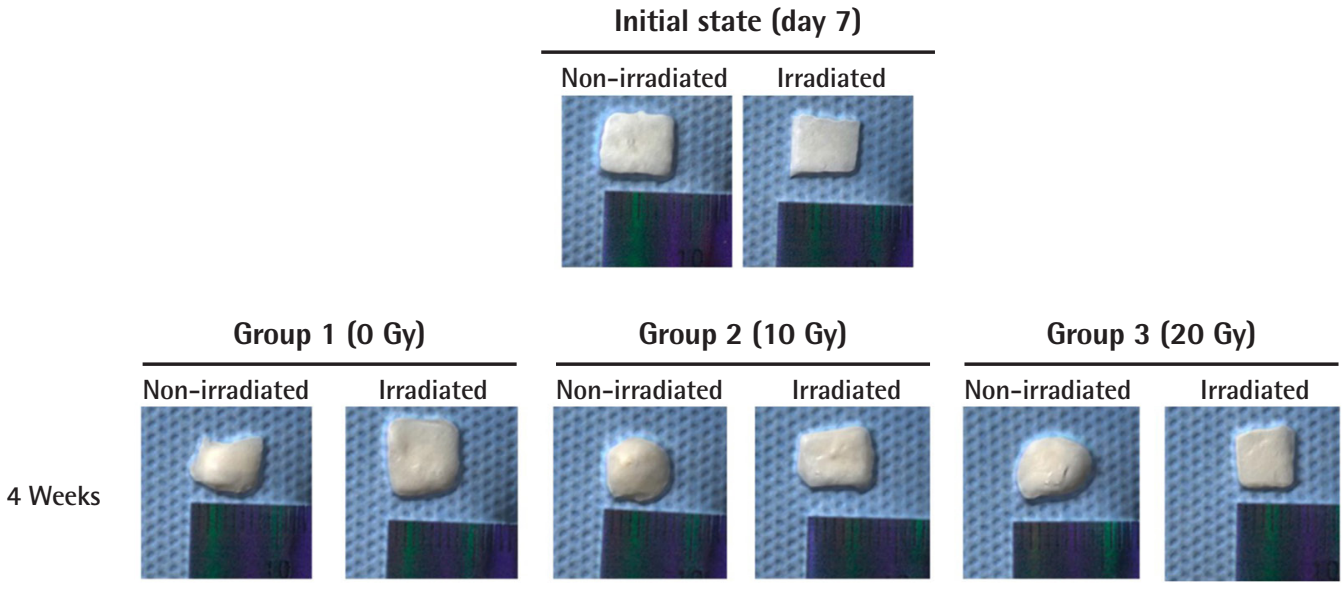

8 Weeks
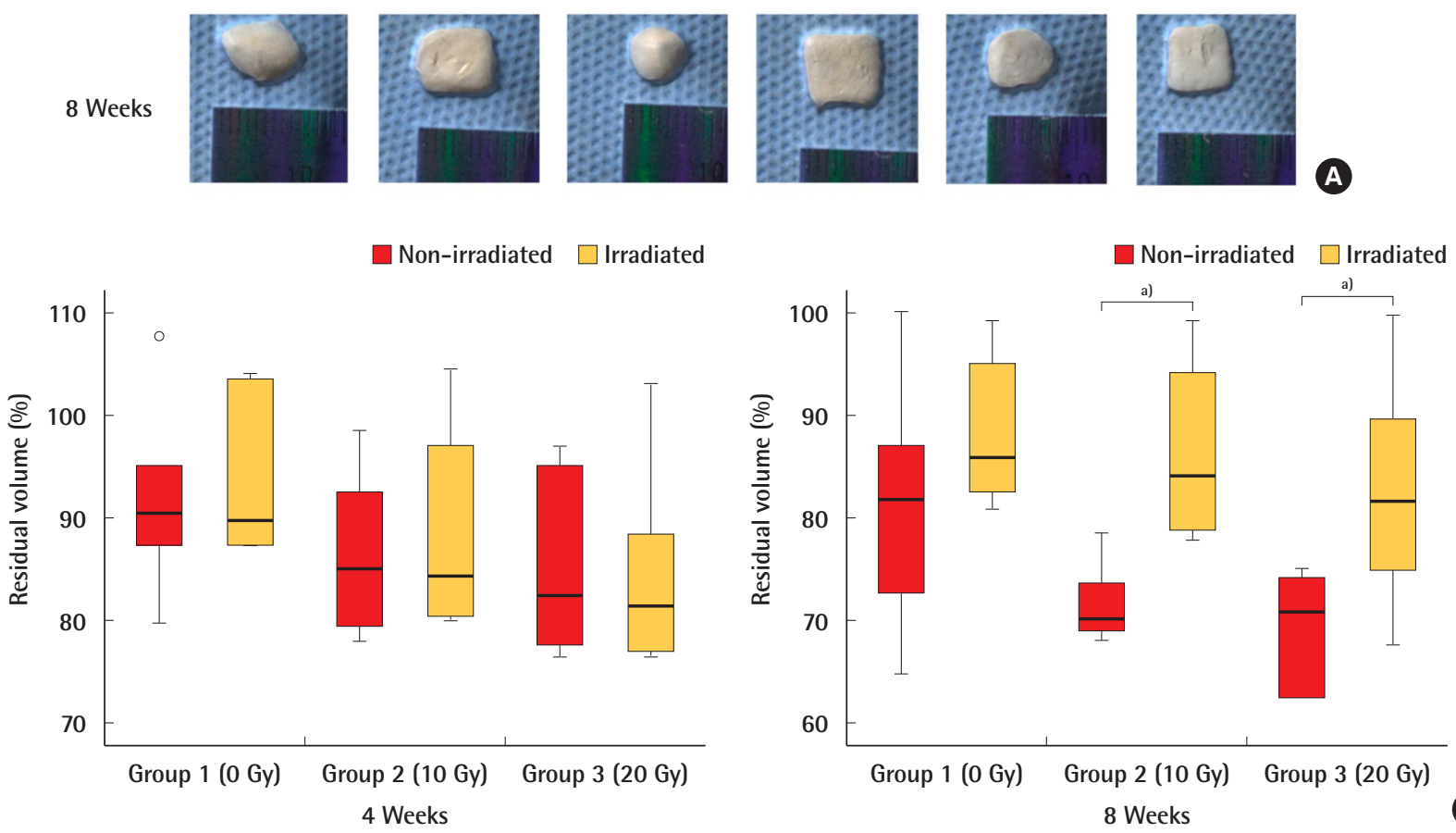

prosthetic-based reconstructions, and it seems that the use of $\mathrm{ADMs}$ to support postmastectomy breast reconstruction is inevitable [11]. The main advantage of ADM use is having greater control over the mastectomy space and the prosthetic device, which facilitates immediate one- and two-stage breast reconstructions. ADM use has been reported to decrease inferior pole rippling and contour abnormalities, improve inferolateral pole coverage, avoid musculature elevation, and improve aesthetic outcomes [2,12]. Furthermore, many animal studies and in vivo studies have suggested that ADM may even slow the progression of capsule formation due to its ability to attenuate the in- flammatory response to a foreign body [13]. Kim et al. [14] reported that the use of ADMs reduced myofibroblasts, vascularity, fibroblasts, and the endothelial-mesenchymal transition in capsule tissues and decreased the quantity of macrophages present, as well as TGF- $\beta 1$ and platelet-derived growth factor subunit B expression. Sbitany et al. [3] compared the results of 50 patients who underwent $\mathrm{ADM}$ reconstruction to those of $50 \mathrm{pa}-$ tients who underwent treatment with a complete submuscular tissue expander or implant placement. No statistically significant difference in complications was found between the two groups. However, reviews have shown that the use of $\mathrm{ADMs}$ increased 


\section{Fig. 3. Cell incorporation of $A D M$}

A comparison of the nucleus counts of cells in acellular dermal matrices (ADMs) showed no significant difference in the number of incorporated cells between irradiated and non-irradiated ADMs. (A) H\&E staining of the ADMs. (B) Quantification of cell incorporation.
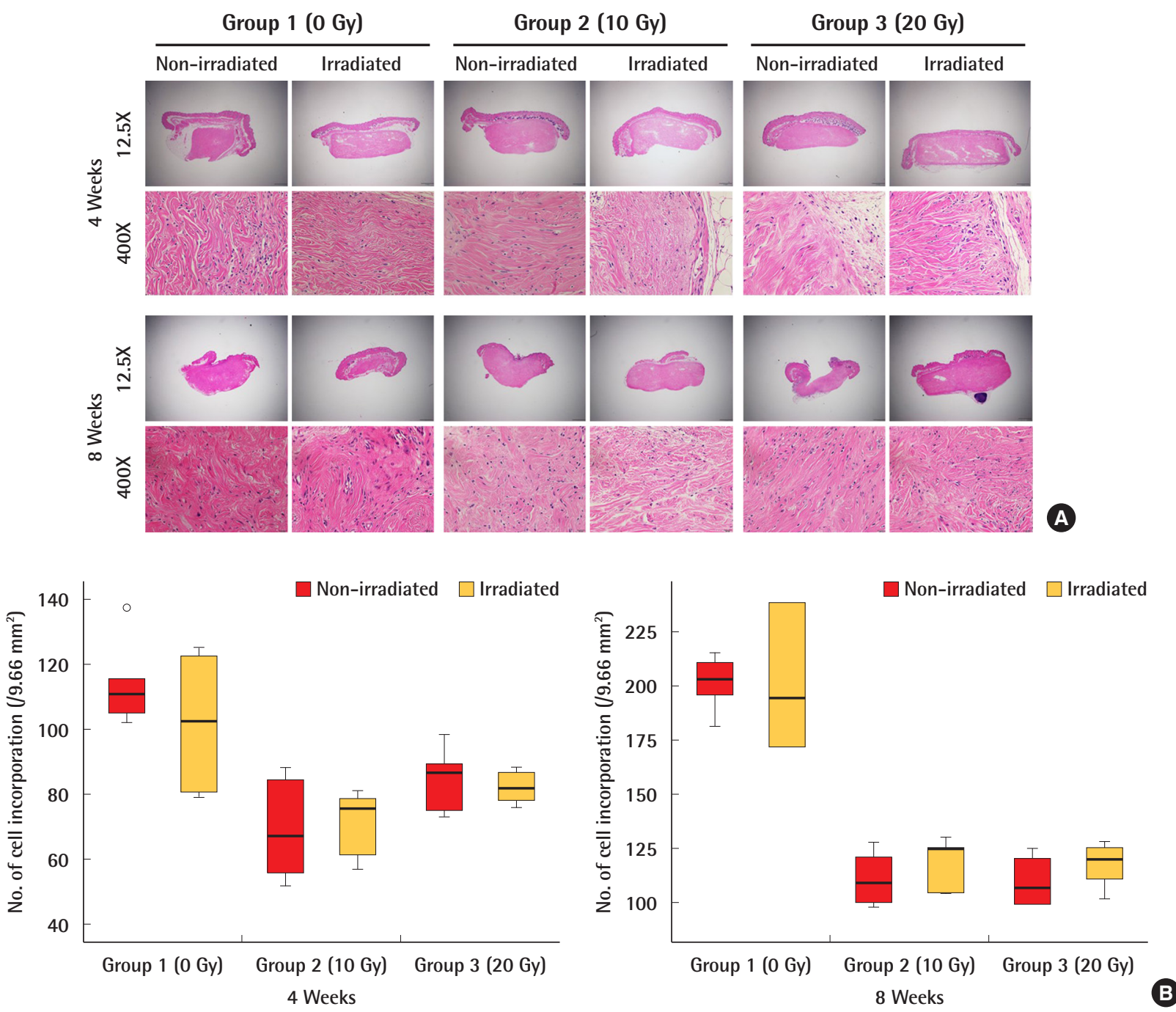

the baseline complication rate for breast reconstruction, which is around $2.5 \%$ [15]. ADM use was found to be associated with higher rates of seroma, infection, and flap necrosis than were found in similar implant reconstructions without ADM [16].

Since $\mathrm{ADMs}$ in breast reconstruction have become an essential option, it is natural that efforts are now being made to improve postoperative outcomes and to reduce complications. Multiple studies have sought to clarify individual variables that may lead to increased complication rates when $\mathrm{ADM}$ is used, such as overweight/obesity, older age, and axillary dissection $[17,18]$. A possible explanation for the finding of a higher risk of infection with $\mathrm{ADM}$ use in breast reconstruction is that $\mathrm{ADM}$ may provide a space for bacteria to reproduce [7]. Thus, it is not surprising that researchers' and clinicians' interest has been drawn to the method of ADM processing (sterile versus aseptic).

Aseptic processing involves refining cells or tissue using techniques to minimize or prevent contamination with microorganisms from the environment, equipment, or processing personnel, according to the definition of the American Association of Tissue Banks [7]. No sterility assurance level is associated with aseptic processing because it does not involve a validated terminal sterilization process. The US Food and Drug Administration defines sterile tissue as tissue that has undergone a terminal sterilization process validated to a sterility assurance level of $10^{-6}$. Thus, to be sterile, an ADM must undergo a validated terminal sterilization process following initial cleansing and decellularization, such as treatment with gamma radiation, electron beam radiation, or chemical sterilization solutions. 


\section{Fig. 4. Expression of $\alpha-S M A$ in ADM}

Myofibroblasts, which play a central role in tissue fibrosis, were not significantly different between irradiated and non-irradiated acellular dermal matrices (ADMs). (A) $\alpha$-Smooth muscle actin ( $\alpha$-SMA) immunostained myofibroblasts (brown staining) of ADMs. (B) Quantification of $\alpha$-SMA positive cells as percent area.
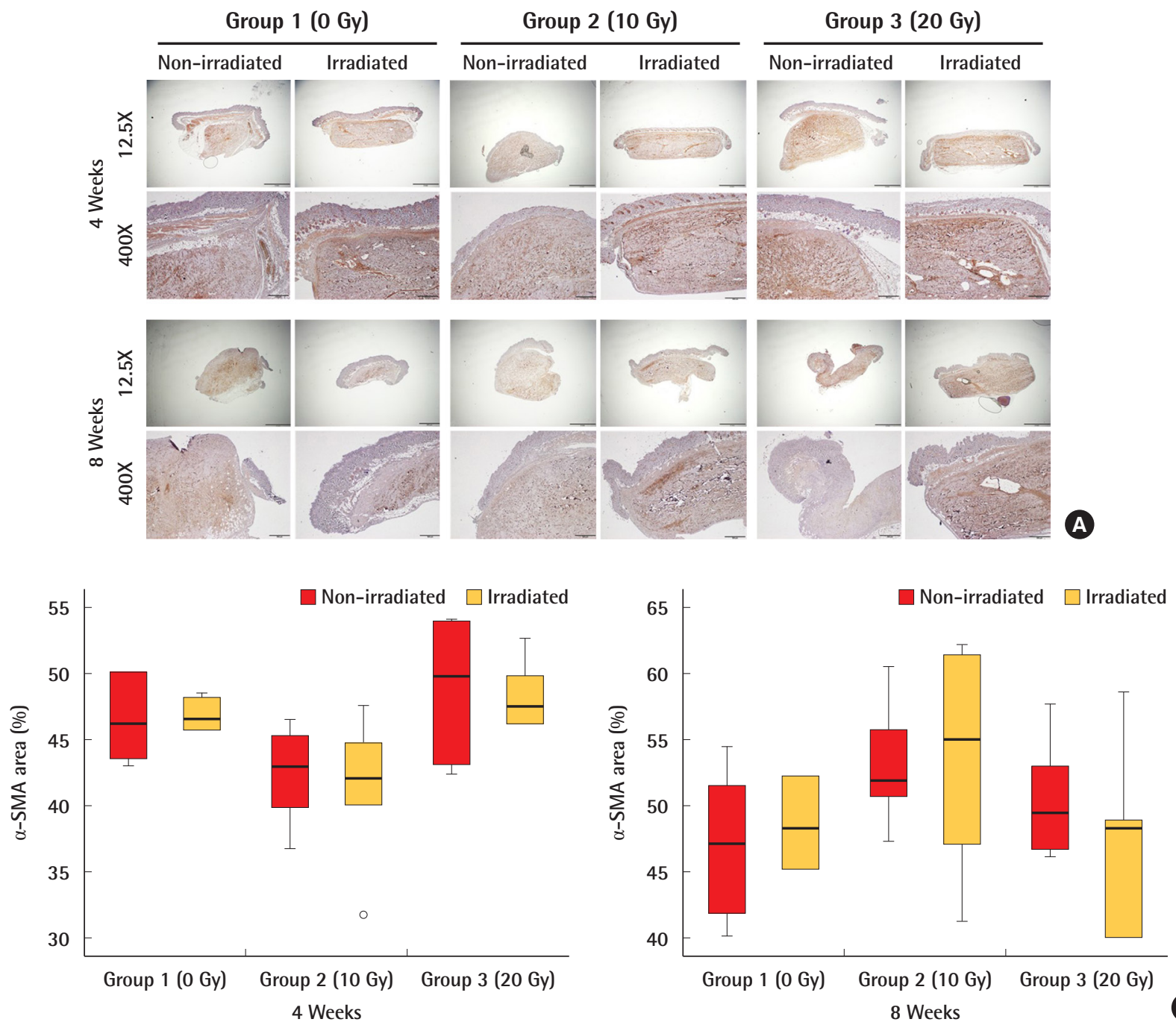

It would be logical to assume that aseptic ADM would be associated with higher rates of complications, especially infection. However, limited studies have compared sterile ADMs to aseptic ADMs in breast reconstruction, with mixed results. Buseman et al. [19] reported significantly higher seroma rates in the sterile AlloDerm ready-to-use (RTU; LifeCell Corporation, Branchburg, NJ, USA) group $(\mathrm{P}=0.003)$ than in the aseptic AlloDerm freeze-dried (FD; LifeCell Corporation) group, but no differences in infection rates. In a similar context, in a prospective study by Klein et al. [20], ADM samples from the first stage of tissue expander-based immediate breast reconstruction were cultured. No growth was found in any cultures, leading the authors to question the need for terminal sterilization of ADM.

However Weichman et al. [2] reported that when comparing reconstructions with sterile $\mathrm{ADMs}$ (AlloDerm RTU) to those with aseptic ADMs (AlloDerm FD), the patients who received sterile $\mathrm{ADMs}$ had a lower risk of overall infection $(\mathrm{P}=0.069)$ and need for explantation $(\mathrm{P}=0.147)$. Venturi et al. [21] also reported that sterile human $\mathrm{ADM}$ could provide reliable matrix incorporation with a low complication rate. Sterilization was not found to have a negative impact on graft integration. In their prospective study, the infection and seroma rates were favorable and comparable to those in previous studies of aseptic ADMs. In light of the above findings, the impact of $\mathrm{ADM}$ processing techniques on postoperative morbidity in patients who undergo implant-based breast reconstruction remains unclear.

Furthermore, no studies have compared ADMs made with different processing techniques in patients undergoing radio- 


\section{Fig. 5. CTGF gene expression level in ADM}

Reverse-transcription polymerase chain reaction results also indicated that the level of mRNA of connective tissue growth factor (CTGF), an essential mediator of fibrosis, was not significantly different between irradiated and non-irradiated acellular dermal matrices (ADMs).
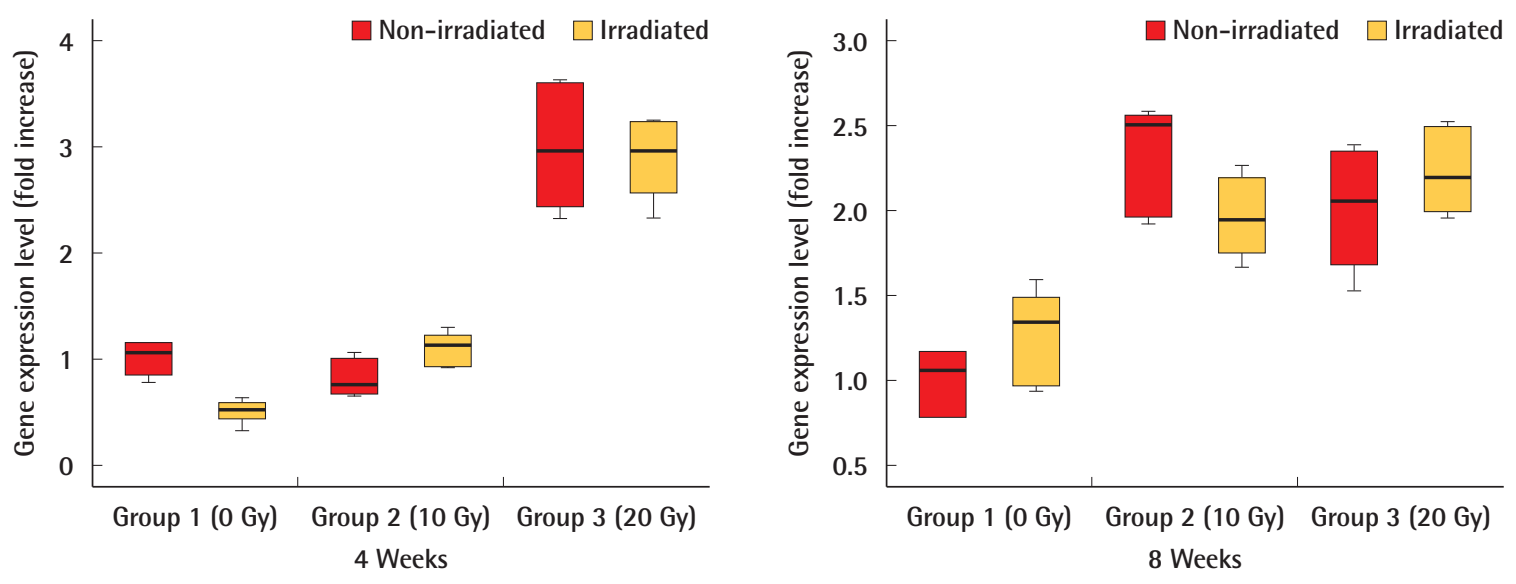

\section{Fig. 6. Dermal thickness}

The dermal thickness covering the acellular dermal matrix (ADM) was significantly greater in the non-irradiated ADMs than in the irradiated ADMs in group 3 (20 Gy) after 4 and 8 weeks. (A) Masson trichome staining of the dermis covering the ADM. The intensity of Masson trichrome staining (in blue) depends on the content of collagen fibers in the tissue. Yellow arrows indicate bundles of collagen that were stained with deeper blue. (B) Quantification of the thickness of the dermis. ${ }^{\text {a) }} P<0.05$.
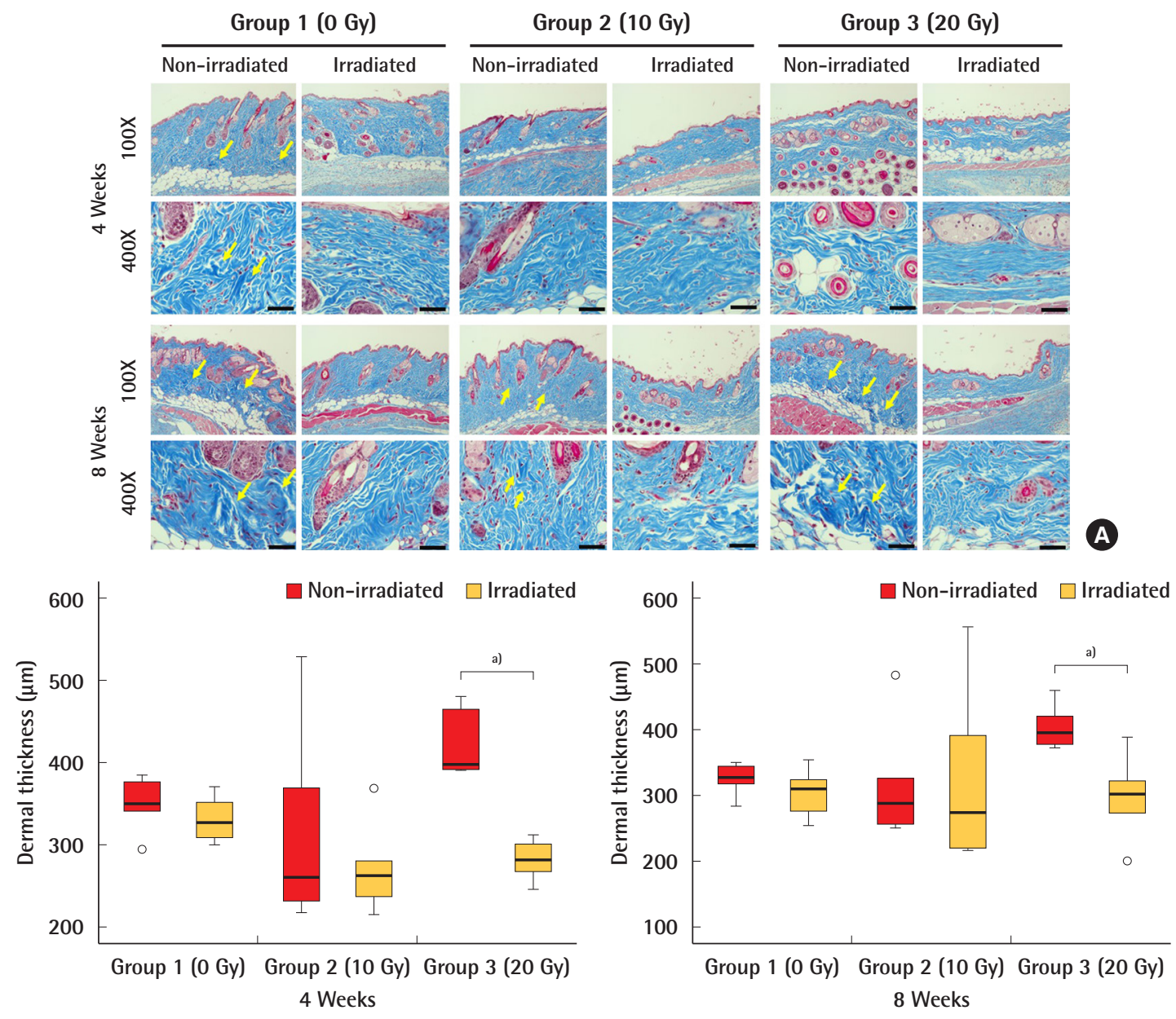


\section{Fig. 7. CD3 staining of the dermis}

Immunohistochemical analyses of inflammation using CD3 as a biomarker showed that there was a significantly smaller extent of inflammation in irradiated acellular dermal matrices (ADMs) than in non-irradiated ADMs. (A) CD3 positive cells in irradiated and non-irradiated ADMs. (B) Quantification of CD3. ${ }^{\text {al }} \mathrm{P}<0.05$.
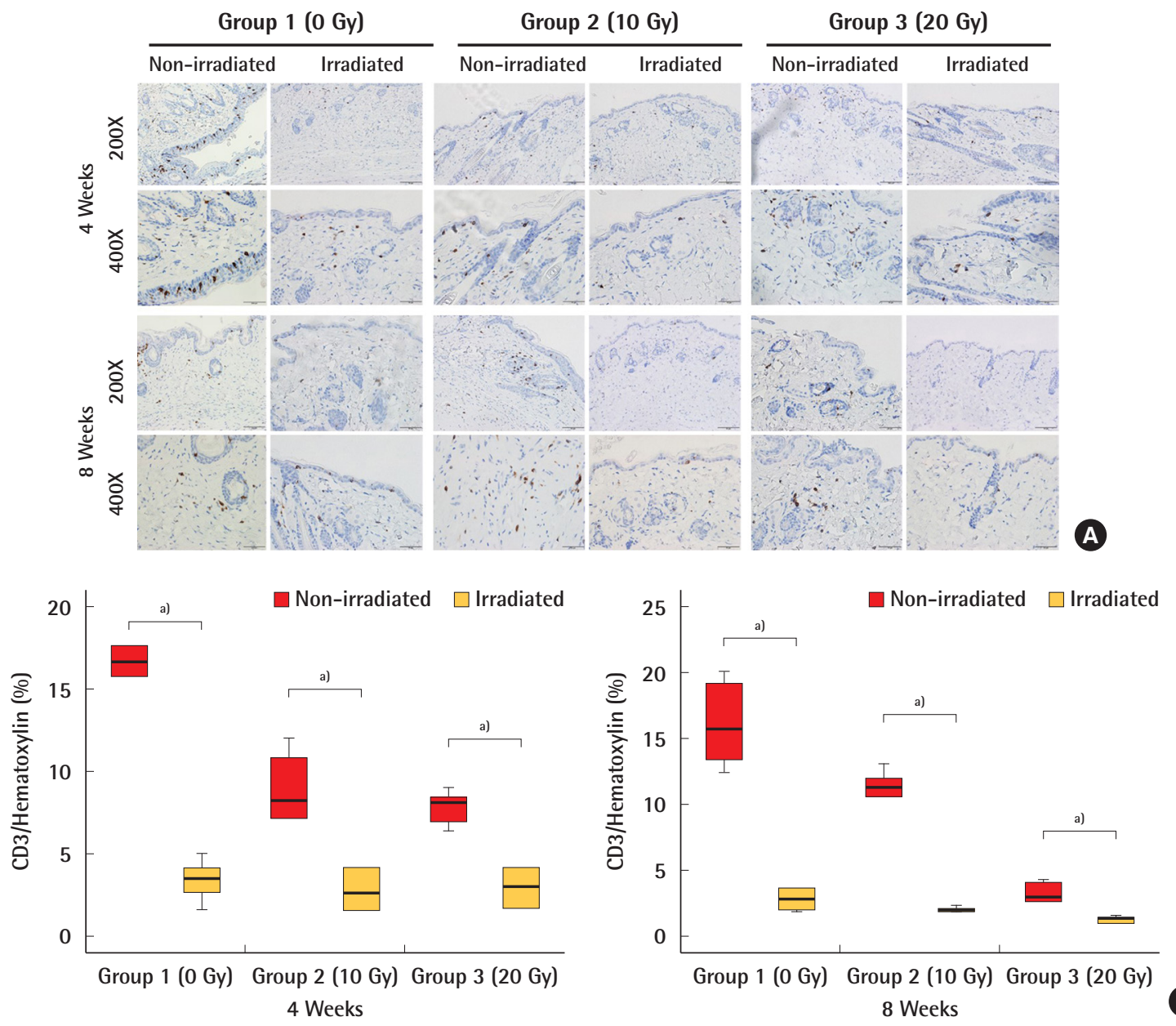

therapy. As far as we know, recent studies have only investigated the effects of radiation on $\mathrm{ADM}$ without considering its sterility. Irradiation is well known to have a negative impact, especially for patients who undergo tissue expander or implant-based breast reconstruction [22]. Whether the addition of $\mathrm{ADM}$ is protective or harmful in the setting of radiotherapy is equivocal. Moyer et al. [23] examined the clinical impact of radiotherapy on $\mathrm{ADM}$ integration to determine whether $\mathrm{ADM}$ plays a protective role against increased fibrosis and capsular formation. They concluded that ADM limits the elastosis and chronic inflammation observed in implant-based breast reconstruction with radiotherapy. Similarly, in a review article of 13 journal articles, Clemens and Kronowitz [24] concluded that the use of $\mathrm{ADM}$ in implant-based breast reconstruction in patients undergoing radiation therapy did not result in higher infection or overall complication rates, or prevent bioprosthetic mesh incor- poration. However, Salzberg et al. [22] found a four-fold higher complication rate in irradiated than in non-irradiated breasts, even with the use of ADMs. The use of ADMs in the context of radiotherapy remains controversial.

The results of $3 \mathrm{D}$ photography showed less volume change in irradiated $\mathrm{ADM}$ s than in non-irradiated $\mathrm{ADM}$ s. A possible reason may be the cross-linking of collagen biomaterials. The crosslinking process increases interfibrillar and intrafibrillar bonds, resulting in increased durability, strength, and stabilization of the graft. When creating irradiated $\mathrm{ADM}$, gamma radiation induces collagen cross-linking, making the ADM more durable. An evaluation of cell incorporation, based on the expression of $\alpha$-SMA and CD3 to assess fibrosis in grafted ADM, showed no noticeable differences in fibrosis between irradiated $\mathrm{ADMs}$ and non-irradiated $\mathrm{ADMs}$ under radiation. Based on this result, applying additional radiation to $\mathrm{ADMs}$ that have already under- 
gone sterilization with gamma radiation did not affect fibrosis.

Numerous studies previously reported that the presence of ADM seems to attenuate fibrosis in humans [13]. In this study, dermal thickness was measured to quantify the amount of fibrosis in the host dermis, and significantly less thickness was found in the irradiated $\mathrm{ADMs}$. Since an inflammatory reaction is one of the critical causes of fibrosis, CD3, an inflammatory marker, was analyzed. Lower percentages of stained CD3 were found in irradiated ADMs in all groups under different external radiation intensities. However, the results for group 2 (10 Gy) show that the amount of CD3 staining does not directly reflect the dermal thickness. Other histological changes than the inflammatory response that are involved in the process of fibrosis should be considered. Additionally, various inflammation markers other than CD3 should also be tested to reach a more solid conclusion.

This study's limitations include its small sample size and single endpoint, which precluded an experimental comparison of changes in a dynamic biological environment. Furthermore, although $\mathrm{ADM}$ processing with gamma radiation is of great concern in terms of sterility and infection problems, this study only dealt with the volume and shape of the ADM and dermis. Lastly, further clinical research is required to discover more about the durability of irradiated ADMs and their function in humans.

This is the first experimental study to compare irradiated and non-irradiated ADMs with radiation exposure in animals. It is well known that postmastectomy radiation therapy can have adverse effects on the aesthetic outcomes of immediate breast reconstruction. However, the effect of the sterility of ADM on the final results after radiation therapy is not known. In this mouse model, the irradiated ADM was more durable, with a smaller volume decrease, than the non-irradiated ADM. The irradiated $\mathrm{ADM}$ also showed less fibrosis, with the deposition of fewer collagen fibers in the dermis, and less inflammation under radiation exposure. This study will provide a basis for future studies to determine a more effective way of manufacturing durable ADM more capable of preserving its shape and volume after radiotherapy.

\section{NOTES}

\section{Conflict of interest}

No potential conflict of interest relevant to this article was reported.

\section{Ethical approval}

The study was approved by the Animal Care and Utilization Committee of the Korea Institute of Radiological and Medical Sciences (approval No. KIRAMS 2018-0078).

\section{Author contribution}

Conceptualization: SJ Woo, US Jin. Data curation: SJ Woo, US Jin. Formal analysis: US Jin. Methodology: SJ Woo. Project administration: SJ Woo. Writing - original draft: SJ Woo. Writing review \& editing: SJ Woo, JH Ha, US Jin

\section{ORCID}

Soo Jin Woo https://orcid.org/0000-0003-3476-8133

Jeong Hyun Ha

Ung Sik Jin https://orcid.org/0000-0002-0927-4185 https://orcid.org/0000-0001-5096-6207

\section{REFERENCES}

1. Israeli R. Complications of acellular dermal matrices in breast surgery. Plast Reconstr Surg 2012;130(5 Suppl 2): 159S-172S.

2. Weichman KE, Wilson SC, Saadeh PB, et al. Sterile "readyto-use" AlloDerm decreases postoperative infectious complications in patients undergoing immediate implant-based breast reconstruction with acellular dermal matrix. Plast Reconstr Surg 2013;132:725-36.

3. Sbitany H, Sandeen SN, Amalfi AN, et al. Acellular dermisassisted prosthetic breast reconstruction versus complete submuscular coverage: a head-to-head comparison of outcomes. Plast Reconstr Surg 2009; 124:1735-40.

4. Israeli R, Feingold RS. Acellular dermal matrix in breast reconstruction in the setting of radiotherapy. Aesthet Surg J 2011;31(7 Suppl):51S-64S.

5. Kim JY, Davila AA, Persing S, et al. A meta-analysis of human acellular dermis and submuscular tissue expander breast reconstruction. Plast Reconstr Surg 2012;129:28-41.

6. Antony AK, McCarthy CM, Cordeiro PG, et al. Acellular human dermis implantation in 153 immediate two-stage tissue expander breast reconstructions: determining the incidence and significant predictors of complications. Plast Reconstr Surg 2010;125:1606-14.

7. Lyons DA, Mendenhall SD, Neumeister MW, et al. Aseptic versus sterile acellular dermal matrices in breast reconstruction: an updated review. Plast Reconstr Surg Glob Open 2016;4:e823.

8. Klein GM, Nasser AE, Phillips BT, et al. Is sterile better than aseptic? Comparing the microbiology of acellular dermal matrices. Plast Reconstr Surg Glob Open 2016;4:e761.

9. Yuen JC, Yue CJ, Erickson SW, et al. Comparison between freeze-dried and ready-to-use AlloDerm in alloplastic breast reconstruction. Plast Reconstr Surg Glob Open 2014;2: e119.

10. Kronowitz SJ. Current status of implant-based breast recon- 
struction in patients receiving postmastectomy radiation therapy. Plast Reconstr Surg 2012;130:513e-523e.

11. Phillips BT, Bishawi M, Dagum AB, et al. A systematic review of infection rates and associated antibiotic duration in acellular dermal matrix breast reconstruction. Eplasty 2014; 14:e42.

12. JoAnna Nguyen T, Carey JN, Wong AK. Use of human acellular dermal matrix in implant- based breast reconstruction: evaluating the evidence. J Plast Reconstr Aesthet Surg 2011; 64:1553-61.

13. Basu CB, Leong M, Hicks MJ. Acellular cadaveric dermis decreases the inflammatory response in capsule formation in reconstructive breast surgery. Plast Reconstr Surg 2010; 126:1842-7.

14. Kim IK, Park SO, Chang H, et al. Inhibition mechanism of acellular dermal matrix on capsule formation in expanderimplant breast reconstruction after postmastectomy radiotherapy. Ann Surg Oncol 2018;25:2279-87.

15. Cordeiro PG, McCarthy CM. A single surgeon's 12-year experience with tissue expander/implant breast reconstruction: part I. a prospective analysis of early complications. Plast Reconstr Surg 2006;118:825-31.

16. Hoppe IC, Yueh JH, Wei CH, et al. Complications following expander/implant breast reconstruction utilizing acellular dermal matrix: a systematic review and meta-analysis. Eplasty $2011 ; 11: \mathrm{e} 40$.

17. Vu MM, Kim JY. Current opinions on indications and algorithms for acellular dermal matrix use in primary prosthetic breast reconstruction. Gland Surg 2015;4:195-203.
18. Winocour S, Martinez-Jorge J, Habermann E, et al. Early surgical site infection following tissue expander breast reconstruction with or without acellular dermal matrix: national benchmarking using National Surgical Quality Improvement Program. Arch Plast Surg 2015;42:194-200.

19. Buseman J, Wong L, Kemper P, et al. Comparison of sterile versus nonsterile acellular dermal matrices for breast reconstruction. Ann Plast Surg 2013;70:497-9.

20. Klein GM, Singh G, Marquez J, et al. Acellular dermal matrix sterility: does it affect microbial and clinical outcomes following implantation? Plast Reconstr Surg Glob Open 2019; 7:e2355.

21. Venturi ML, Mesbahi AN, Boehmler JH 4th, et al. Evaluating sterile human acellular dermal matrix in immediate expander-based breast reconstruction: a multicenter, prospective, cohort study. Plast Reconstr Surg 2013;131:9e-18e.

22. Salzberg CA, Ashikari AY, Koch RM, et al. An 8-year experience of direct-to-implant immediate breast reconstruction using human acellular dermal matrix (AlloDerm). Plast Reconstr Surg 2011;127:514-24.

23. Moyer HR, Pinell-White X, Losken A. The effect of radiation on acellular dermal matrix and capsule formation in breast reconstruction: clinical outcomes and histologic analysis. Plast Reconstr Surg 2014;133:214-21.

24. Clemens MW, Kronowitz SJ. Acellular dermal matrix in irradiated tissue expander/implant-based breast reconstruction: evidence-based review. Plast Reconstr Surg 2012;130 (5 Suppl 2):27S-34S. 